\title{
МЕТЕОРОЛОГИЯ, КЛИМАТОЛОГИЯ
}

УДК 577.3

\section{ВЛИЯНИЕ КЛИМАТА И ПОГОДЫ НА САМОЧУВСТВИЕ И ЗДОРОВЬЕ ЛЮДЕЙ. СОВРЕМЕННЫЕ ПРЕДСТАВЛЕНИЯ}

\author{
Т.А. Зенченко ${ }^{1,2}$, Т.К. Бреус ${ }^{1}$ \\ ${ }^{1}$ Институт космических исследований РАН, Москва, Россия \\ ${ }^{2}$ Институт теоретической и экспериментальной биофизики РАН, Пущино, Московская область, Россия
}

Приводится анализ и оценка работ по уже установленным особенностям метеоэффекта, таким как нелинейность, немонотонность, временная изменчивость, относительность амплитуды, наличие временного лага и др. Обосновывается необходимость, наряду с традиционным популяционным подходом, более широкого применения индивидуального метода оценки метеочувствительности. Подобный анализ необходим для последующего синтеза первичных результатов и построения глобальной рабочей модели системы «человек - атмосфера».

Ключевые слова: биометеорология, метеочувствительность, популяиионная статистика, индивидуальный подход, артериальное давление, сердечно-сосудистая система.

\section{Введение}

Биометеорология - наука о реакции организма человека на действие факторов погоды - насчитывает много столетий. Еще в Древней Греции Гиппократ впервые отметил сезонное чередование обострений различных недугов. В своих книгах об эпидемических заболеваниях описание каждой болезни он начинает с влияния на нее метеорологических условий.

В середине ХХ в. Д. Ассман [Ассман, 1966] сформулировал основную проблему биоклиматологии того времени: между многочисленными метеорологическими явлениями и определенными биологическими реакциями обнаруживаются статистически достоверные временные связи, но истинные действующие факторы и механизмы их влияния на организм до сих пор еще не выяснены, поскольку многочисленные факты не укладываются ни в одну из умозрительных схем.

За прошедшие с момента появления этой книги 60 лет существенно улучшились скорость и возможности получения самой свежей информации о результатах коллег, значительно расширился математический аппарат для многопараметрического анализа больших объемов данных, выросли вычислительные мощности и возможности компьютерного моделирования.

Однако при всех перечисленных качественных прорывах задача построения глобальной рабочей модели метеочувствительности остается далекой от разрешения. Основной проблемой является значительная вариабельность эффекта: даже при видимой близости начальных условий задачи и применяемых методов анализа результаты разных групп исследователей оказываются зачастую качественно различными.

В 70-е гг. XX в. перечень внешних факторов, традиционно рассматриваемых как биотропные, был расширен: к числу атмосферных добавились факторы космической погоды, что еще более усложнило изучаемую систему. В дальнейшем было показано [Бреус и др., 2010, 2016; Ожередов и др., 2010; Зенченко, 2012; Gurfinkel et al., 2012], что изучение отдельно эффектов солнечной и геомагнитной активности (без учета метеоэффектов) методологически некорректно, поскольку влияние этих двух групп факторов имеет мишенью одни и те же системы организма и оказывает сочетанное действие. Однако в данной работе мы поставили целью проанализировать исследования, посвященные влиянию только метеорологических факторов, но оставили за границами рассмотрения исследования, посвященные действию факторов космической погоды.

Такую постановку задачи можно допустить из-за различий частотного профиля реакции организма человека на действие атмосферных и гелиогеофизических факторов. В работах [Бреус и др., 2010, 2016; Ожередов и др., 2010; Зенченко, 2012; Gurfinkel et al., 2012] было показано, что метеоэффект является «медленным» относительно магнитоэффекта: в первом случае мы наблюдаем синхронизацию волн длительностью от нескольких дней до двух недель, а во втором - резкие пикообразные всплески в течение 1-2 сут. Следовательно, вклад этих двух групп внешних факторов оказывается несимметричным, и воздействие метеофакторов на организм может рас- 
сматриваться без учета влияния магнитоэффекта, но не наоборот.

Таким образом, целью данной работы было проанализировать и систематизировать известные на настоящий момент причины, влияющие на выраженность метеоэффекта, а также обосновать возможные изменения методологического подхода, позволяющие, на наш взгляд, в перспективе преодолеть системный кризис, существующий в биометеорологии.

\section{1. Популяционные статистические исследования}

\section{1. Основные направления исследования метеоэффекта}

Сформулируем основные известные на настоящий момент характеристики метеоэффекта по данным публикаций в основном за последние 20 лет.

Метеотропность (чувствительность организма к погодным факторам) рассматривается в биометеорологии как универсальное свойство всех без исключения биосистем, включающее в себя различные их состояния: от нормы - через напряжение (адаптацию) до патологии и смерти [Андронова и др., 1982].

В настоящее время в биометеорологии существует три основных подхода к сбору и анализу медикобиологических данных: популяционные исследования, лабораторные клинические исследования и индивидуальный мониторинг. У каждого из этих подходов есть свои преимущества и недостатки.

Все работы в области исследования метеоэффекта можно разделить на четыре направления по сочетанию двух критериев: типу метеотропной реакции (адаптационная реакция с возвратом к норме или срыв адаптации) и степени экстремальности действующих внешних факторов (сверхвысокие значения или близкие к среднестатистическим). К экстремальным значениям метеопараметров принято относить очень высокие или очень низкие значения, как правило, 1 или $5 \%$ от общего распределения значений, характерных для данного региона (волны жары и холода). Остальная часть выборки наблюдаемых значений, за исключением экстремальных, рассматривается как фоновые, привычные для организма.

Подавляющее число работ и в прошлом, и в настоящее время относится к исследованию метеопатологического эффекта, т.е. срыву адаптации, обусловленного как экстремальными, так и фоновыми вариациями атмосферных факторов, поскольку социальная значимость таких исследований очевидна. В подавляющем большинстве работ материалами для анализа служат данные о показателях смертности и заболеваемости в определенных популяциях.

Также значительное внимание уделяется изучению процессов адаптации здоровых лиц к экстре- мальным условиям высокогорья или полярных зимовок, а также воздействию волн жары или холода на население в целом (например, [Patrikeeva, Basova, 2018] и другие работы). Здесь материалами для анализа являются результаты лабораторных клинических измерений физиологических показателей.

В то же время влиянию вариаций неэкстремальных факторов на самочувствие практически здоровых людей уделяется, на наш взгляд, очень мало внимания, поскольку актуальность таких исследований неочевидна. Однако это направление, на наш взгляд, является теоретическим и практическим базисом для первых трех. Поскольку здоровые добровольцы обычно набираются в качестве контрольной группы сравнения в каждом исследовании, неучитываемая метеочувствительность их физиологических показателей может значительно искажать выводы, полученные при исследовании заболеваемости и смертности. В этом направлении исследований данными для анализа служат либо клинические измерения, либо результаты длительного индивидуального мониторинга.

\section{2. Экстремальные факторы и госпитализаџия}

В последние два десятилетия теме влияния экстремальных факторов погоды на заболеваемость посвящен ряд обзоров, систематизирующих массивы исследований [Turner et al., 2012; Kenney et al., 2014; Casas et al., 2016], а также множество отдельных исследований в разных климатических зонах [Linares, Díaz, 2008; Lin et al., 2009; Wolf et al., 2009; Bayentin et al., 2010; Magalhães et al., 2011; Williams et al., 2012; Lavigne et al., 2014].

Анализ этих работ позволяет выделить следующие особенности метеоэффекта:

- увеличение числа обращений в больницы провоцируют как экстремально высокие, так и экстремально низкие температуры [Linares, Díaz, 2008; Bayentin et al., 2010, Lavigne et al., 2014];

- непосредственной причиной обращения бывает обострение сердечно-сосудистых осложнений [Kenney et al., 2014; Casas et al., 2016] или респираторных заболеваний [Turner et al., 2012];

- дополнительными факторами риска возникновения метеопатологий служат заболеваемость диабетом 2-го типа и пожилой возраст пациентов, поскольку среди этих групп людей метеопатологический эффект выражен сильнеe [Casas et al., 2016];

- метеопатологический эффект характерен для разных климатических зон, в том числе и районов с относительно мягким климатом. Так, зависимость заболеваемости и смертности от экстремальных температур обнаруживается от прохладной Канады [Bayentin et al., 2010; Lavigne et al., 2014] до жарких районов Португалии [Magalhães et al., 2011] и Ав- 
стралии [Williams et al., 2012]. Географическая широта местности практически не влияет на оценку риска респираторной и сердечно-сосудистой заболеваемости [Turner et al., 2012];

- температурный порог, выше которого кардинально возрастает частота госпитализаций, совпадает с аналогичным порогом по смертности и с 95-м процентилем максимального дневного ряда температур для летних месяцев [Linares, Díaz, 2008];

- время задержки между моментом действия метеофактора и вызванным им ростом заболеваемости может различаться и зависит в том числе и от вида заболевания. Например, при высоких температурах (29$\left.36{ }^{\circ} \mathrm{C}\right)$ рост госпитализаций в Нью-Йорке по поводу простуд происходит синхронно с повышением температуры, по поводу сердечно-сосудистых заболеваний отстает в среднем на пять суток [Lin et al., 2009].

Важно отметить, что среди работ по действию экстремальных факторов на здоровье населения все работы посвящены влиянию только одного атмосферного фактора - приземной температуры воздуха, и нет работ по влиянию атмосферного давления. Практически все они посвящены «краям распределения» - очень высоким температурам летом и очень низким - зимой. Удалось найти только одну статью, [Wolf et al., 2009], в которой показана опасность не экстремальных, а аномальных температур, т.е. увеличение риска инфарктов и коронарных смертей при резких понижениях температуры в течение лета, причем эффект был выражен тем сильнее, чем выше была в этот год среднесезонная температура.

\section{3. Фоновые вариации погоды и госпитализациии}

Исследования роли неэкстремальных вариаций атмосферных факторов на показатели госпитализации, заболеваемости и смертности наиболее широко представлены в литературе.

Условно всю выборку статей можно разбить на три группы по критерию исследуемых метеофакторов:

- только температуры воздуха $\left(\mathrm{T}_{\text {атм }}\right)$ и ее производных (максимальная, перепад в течение суток, средняя за сутки) [Berginer et al., 1989; Armstrong, 2006; Basu, 2009; Bhaskaran et al., 2009; Ye et al., 2012; Basu et al., 2012; Hong et al., 2012; Zhang et al., 2018; Zhao \& Cheng, 2019; Davis et al., 2020; Madaniyazi et al., 2020];

- только атмосферного давления (Р менчивости, но без учета влияния температуры [Dockery et al., 1999; Houck et al., 2005; Azcárate, Mendoza, 2017];

- весь комплекс атмосферных факторов или некоторое их сочетание ( $\mathrm{T}_{\text {атм }}, \mathrm{P}_{\text {атм}}$, относительная влажность воздуха (Relative Humidity, RH), сила ветра и их вариации) [Danet et al., 1999; Feigin et al., 2000; Ebi et al., 2004; Kulakov, Nasonova, 2004; Čulič et al., 2004; Głuszak et al., 2008; Jie et al., 2014; Comelli et al., 2014; Shaposhnikov et al. 2014; Vencloviene et al., 2015; 2018; Ozheredov et al., 2017].

Основные выводы существующих обзорных исследований влияния фоновых вариаций температуры близки к описанным выше выводам для эффектов экстремальных температур:

- как относительно жаркая, так и относительно холодная погода оказывают пагубное влияние на риск возникновения осложнений здоровья [Bhaskaran et al., 2009];

- повышенная температура увеличивает риск смертности в основном от сердечно-сосудистых, респираторных и цереброваскулярных заболеваний [Basu, 2009];

- характеристики эффекта зависят от расы, пола, возраста, места проживания пациента [Ye et al., 2012];

- при попытках сопоставления направления и величины нелинейных эффектов запаздывания авторы работы [Ye et al., 2012] делают вывод, что создать общую картину не удается в силу расхождений результатов в разных работах.

Таким образом, авторы обзоров утверждают многопараметричность эффекта метеочувствительности, систематизировать которую в настоящее время не удается.

Анализ отдельных исследований позволяет добавить еще ряд важных особенностей, характерных для температурного метеоэффекта:

- географическая универсальность эффекта: Израиль [Berginer et al., 1989], Китай [Jie et al., 2014; Zhao, Cheng, 2019], Калифорния [Ebi et al., 2004; Basu et al., 2012], Англия и Уэльс [Armstrong, 2006; Zhang et al., 2018];

- разнонаправленность эффекта в разных исследованиях. В одних работах сообщается о положительной корреляции между значениями температуры и показателями заболеваемости инфарктами [Schwartz et al., 2004; Ebi et al., 2004], инсультами [Berginer et al., 1989], ишемической болезнью сердца, ишемическим инсультом, сердечной аритмией, гипотонией, диабетом [Basu et al., 2012]. В других работах корреляция отрицательна [Danet et al., 1999; Shaposhnikov et al., 2014, Zhao, Cheng, 2019];

- немонотонность зависимости относительного риска сердечно-сосудистых заболеваний (всех в совокупности) от $\mathrm{T}_{\text {атм. }}$ В работе [Jie et al., 2014] сообщается, что кривая зависимости заболеваемости имеет не минимум, а максимум, расположенный при $10^{\circ} \mathrm{C}$, т.е. авторы фиксируют снижение риска заболеваемости при очень высоких и низких температурах;

- важность не только самих значений, но и внутри- и межсуточной вариабельности параметра температуры. В работе [Davis et al., 2020] сделан вывод об опасности очень холодных дней, которые сочетаются с высокой внутрисуточной вариабельностью 
температуры. Исследование [Zhang et al., 2018] также подтверждает важную роль изменчивости $\mathrm{T}_{\text {атм }}$ в динамике смертности (в 10 районах Англии и Уэльса);

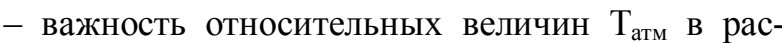
пределениях значений, характерных для данной местности. Так, при сравнении влияния температуры на смертность в шести городах Японии авторы [Ng et al., 2014] делают вывод, что эффекты конкретных городов, основанные на абсолютных изменениях температуры, были более разнородными, чем оценки, основанные на относительных изменениях;

- сезонность выраженности эффекта и его изменчивость во времени на масштабах в несколько лет [Zhang et al., 2018]. Так, доля смертей, обусловленных вариабельностью температуры, в среднем составляет, по оценкам авторов, около $2,5 \%$, и имеет сезонные колебания от 3,08 \% летом до 2,00 \% зимой;

- чувствительность к изменениям $\mathrm{T}_{\text {атм }}$ биохимических показателей крови, таких как сердечнососудистые маркеры и липопротеины [Hong et al., 2012; Madaniyazi et al., 2020]. Hong и соавт. [Hong et al., 2012] утверждают, что изменение знака производной происходит при нулевой температуре: уровень высокочувствительного С-реактивного белка повышался с понижением температуры при минимальных температурах ниже $0{ }^{\circ} \mathrm{C}$, но обнаруживал обратную связь выше. Madaniyazi и соавт. [Madaniyazi et al., 2020] также говорят о нелинейности зависимости профиля липопротеинов, таких как триглицериды, липопротеины низкой и высокой плотности, от температуры. Чувствительность уровня сердечных маркеров к изменениям температуры дает авторам этих работ основания предполагать, что данные биохимические факторы задействованы в формировании метеотропного ответа организма;

- двумерное соотношение температуры и смертности в Лондоне от величины временного лага является нелинейным и сохраняется (но меняет форму) с увеличением задержки как минимум до 14 сут [Armstrong, 2006]. Риск возрастает при нулевом лаге и сохраняется повышенным вплоть до задержки реакции до пяти суток, а также и при температуре от 17 до $29^{\circ} \mathrm{C}$;

- наибольшую метеочувствительность показывают изменения физиологических показателей у пожилых людей как основной группы риска. И хотя в этих работах речь идет еще не о срыве адаптации, как при госпитализациях, но о близком к нему диапазоне напряжения и перенапряжения адаптационных систем организма, границу между которыми обозначить достаточно сложно. Авторы этих исследований сообщают, что при снижении температуры воздуха у пожилых людей наблюдаются значительное повышение показателей артериального давления (АД) [Woodhouse et al., 1993; Alperovitch et al., 2009; Ren et al., 2011; Halonen et al., 2011], а также количе- ства тромбоцитов и концентрации липопротеинов холестерина в сыворотке крови низкой плотности [Hong et al., 2012]. В зимний период по сравнению с летом доля пациентов с АД большим 160/90 мм рт. ст. увеличилась в четыре раза [Woodhouse et al., 1993]. Сообщается о связи вариаций температуры с длительностью сердечного показателя QT [Mehta et al., 2014].

Вторая группа статей, сравнительно немногочисленная, показывает существование значимых корреляций между суточными вариациями $\mathrm{P}_{\text {атм и числом }}$ госпитализаций по поводу острого инфаркта миокарда (ИМ) и инсульта [Houck et al., 2005], а также изменениями АД, частоты сердечных сокращений (ЧСС) и насыщения крови кислородом у больных и пожилых людей [Dockery et al., 1999; Azcárate, Mendoza, 2017].

К сожалению, в двух описанных выше группах исследований рассматривается возможное влияние только одного из атмосферных факторов, либо температуры, либо атмосферного давления. Поэтому из них невозможно однозначно установить, какой из факторов является основным, поскольку их динамика сильно скоррелирована, а характер корреляции кардинально зависит от географической широты точки наблюдения

Так, в работе [Зенченко и др., 2009] была изучена динамика парной взаимосвязи показателей атмосферного давления, относительной влажности и температуры воздуха на протяжении 1999-2010 гг. для трех климатических регионов (рис. 1). Из рис. 1, $a$ видно, что параметры $\mathrm{T}_{\text {атм и }} \mathrm{P}_{\text {атм }}$ тесно связаны в южных широтах на протяжении всего года и независимы в средних и высоких широтах. В то же время если летом вариации температуры и относительной влажности отрицательно скоррелированы во всех географических точках, то в зимний период в северных широтах эти параметры имеют положительную корреляцию, а в южных районах статистически значимой связи между ними нет (рис. $1, b$ ).

В частности, за рамками описанных выше исследований остается вопрос о соотношении вкладов различных атмосферных факторов в метеотропный эффект, о необходимости их комплексного учета, о поиске многомерных областей значений, в которых метеотропный эффект максимален.

Эти вопросы обсуждаются в третьей группе работ, где анализируются возможные медицинские последствия вариаций одновременно нескольких атмосферных факторов либо их комплексов.

Наиболее популярный набор параметров включает обычно $\mathrm{T}_{\text {атм }}, \mathrm{P}_{\text {атм }}$, относительную влажность воздуха, их межсуточную изменчивость и показатели геомагнитной активности [Danet et al., 1999; Feigin et al., 2000; Magalhães et al., 2011; Jie et al., 2014; Comelli et al., 2014; Shaposhnikov et al., 2014]. В некоторых работах исследуется также влияние силы ветра, осадков, 
параметров состояния гелиосферы (компоненты межпланетного магнитного поля, космических лучей, солнечного ветра) [Kulakov, Nasonova, 2004; Čulič et al., 2004; Głuszak et al., 2008; Vencloviene et al., 2015, 2018] или что-то географически специфическое, например, влияние Эль-Ниньо [Ebi et al., 2004]. $a$

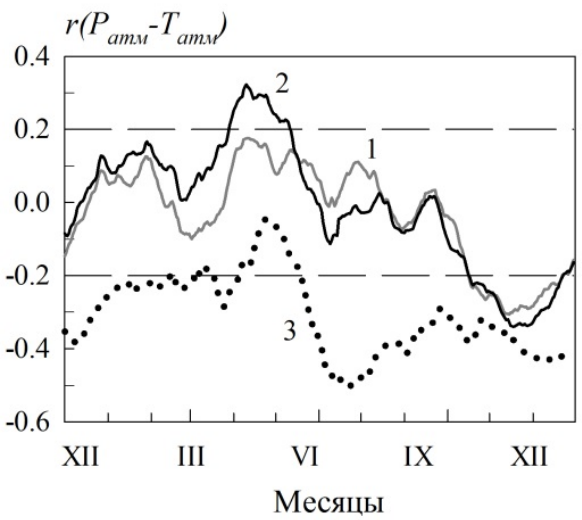

b

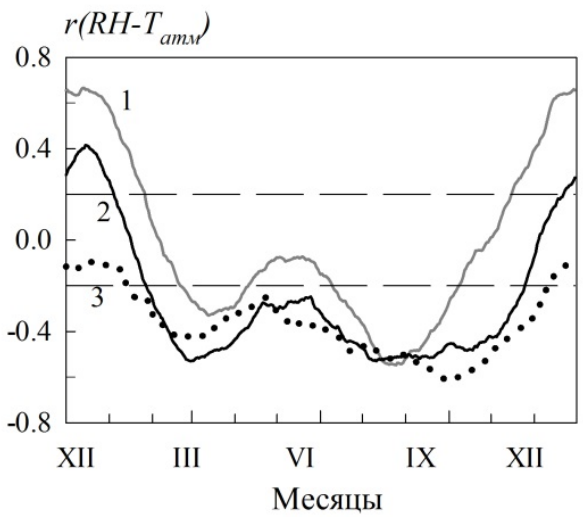

Рис. 1. Усредненная годовая динамика функций корреляции, вычисленных в скользящем окне, для пар показателей $P_{\text {атм }}-\mathrm{T}_{\text {атм }}(a)$ и $\mathrm{T}_{\text {атм }} \mathrm{RH}(\boldsymbol{b})$ для Сыктывкара (1), Москвы (2) и Симферополя (3) Пунктирами показаны границы $5 \%$ уровня статистической значимости

Fig. 1. Annual dynamics of atmospheric factors correlation - (a) $P_{\mathrm{atm}}$ and $T_{\mathrm{atm}}$; (b) $R H$ and $T_{\mathrm{atm}}$ : Syktyvkar (1), Moscow (2) and Simferopol (3)

The dotted lines show the boundaries of the 5\% level of statistical significance

Основным медицинским показателем являются данные обращений в больницы по поводу обострения сердечно-сосудистых заболеваний: гипертонической болезни, инфарктов и инсультов. В некоторых работах рассматриваются более специфические нозологии, такие как случаи острой фибрилляции предсердий [Comelli et al., 2014], желудочковой тахикардии [Čulič et al., 2004], мерцательной аритмии [Głuszak et al., 2008].

Большинство исследований формулирует цель работы традиционно: выявить случаи и характеристики статистической зависимости распределения эпидемиологических данных от вариаций атмосферных факторов. Однако при ответе на этот вопрос наблюдаются существенные расхождения полученных результатов.

Например, работы [Danet et al., 1999; Shaposhnikov et al., 2014] можно рассматривать как близкие по исходным данным и ряду входных параметров. Их авторы исследуют заболеваемость в достаточно близких по климату географических районах: на севере Франции (50' с.ш.) и в Москве (56' с.ш.) соответственно. Выводы этих работ согласуются в ключевых положениях: снижение числа инфарктов миокарда с возрастанием температуры (во всем диапазоне исследованных значений $\mathrm{T}_{\text {атм}}$ ) и U-образной (немонотонной) зависимости от значений $\mathrm{P}_{\text {атм. }}$.

Однако в деталях обнаруживаются различия.
Во-первых, различается положение минимума на кривой зависимости заболеваемости от $\mathrm{P}_{\text {атм. }}$ В первой работе положение минимума указывается при $\mathrm{P}_{\text {атм }}=1016$ мбар, во второй - приблизительно 995 мбар. Однако данное расхождение конкретных величин может быть обусловлено различием среднесезонных распределений атмосферного давления в этих географических локализациях (Лилль - высота над уровнем моря 17-27 м, Москва - около 130 м).

Во-вторых, качественно различается форма зависимости числа ИМ от изменений температуры. Так, в работе [Danet et al., 1999] говорят о линейном снижении, однако необходимо заметить, что на соответствующем рисунке (см. рис. 1), виден излом примерно на $17^{\circ} \mathrm{C}$ : выше этой температуры значения числа ИМ начинают расти. Линейное снижение получается у авторов только в результате ограниченности степени выбранного уравнения регрессии.

В работе [Shaposhnikov et al., 2014] кривая зави-

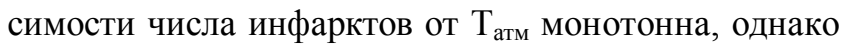
имеется отчетливый излом, отмеченный авторами: практически горизонтальная линия при отрицательных температурах и значительное снижение при положительных.

Инсульты в работе [Danet et al., 1999] не рассматривались. В статье [Shaposhnikov et al., 2014] авторы сообщают о линейном характере роста числа инсультов с возрастанием температуры во всем диапазоне исследованных температур, но также о дву- 
кратном возрастании риска во время холодовых волн. В других работах встречаются выводы о возрастании числа инсультов как при понижении температуры воздуха [Feigin et al., 2000; Magalhães et al., 2011], так и при ее возрастании [Ebi et al., 2004; Feigin et al., 2000; Magalhães et al., 2011; Mostofsky et al., 2014] также отмечают наибольшую опасность именно низких температур для развития инсультов.

Таким образом, при относительной близости ряда входных параметров разных исследований их результаты различаются как количественно, так и в некоторых аспектах - качественно.

В целом же мнение о доминировании роли приземной температуры воздуха как наиболее биотропном факторе преобладают над другими заключениями.

Можно отдельно выделить ряд работ, посвященных метеообусловленности серьезных нарушений сердечного ритма [Comelli et al., 2014] свидетельствует о линейной корреляции числа случаев острой фибрилляции предсердий и температуры воздуха; [Głuszak et al., 2008] при исследовании случаев мерцательной аритмии выделяют значительное влияние холодного фронта и окклюзии типа холодного фронта [Čulič et al., 2004]; в качестве предикторов случаев желудочковой тахикардии называют U-образный паттерн скорости ветра (очень слабый или очень сильный), относительную влажность воздуха, атмосферное давление и эмоциональное расстройство. Таким образом, в этой группе исследований наблюдается многообразие комбинаций метеофакторов, однако с преобладающей ролью $\mathrm{T}_{\text {атм }}$.

В ряде работ (пока немногочисленных) ставится более сложная задача: при каких значениях комбинаций атмосферных параметров наиболее выражен эффект влияния факторов космической погоды на уровни заболеваемости и смертности. Текущие значения метеопоказателей здесь рассматриваются как параметры, а значения геомагнитных индексов - как входные переменные, подлежащие анализу.

Правомочность такой постановки задачи обсуждалась выше во введении к данной статье и обусловлена различием скоростей ответа организма на вариации атмосферных и геомагнитных факторов. К работам с такой постановкой вопроса относятся [Vencloviene et al., 2015; Ozheredov et al., 2017], в которых показано, что выраженность эффекта магниточувствительности (в данном случае - зависимость показателей АД от геомагнитной активности) зависит от текущих значений атмосферных факторов.

\section{4. Вивводы и обсуждение}

Таким образом, популяционные исследования влияния вариаций атмосферных факторов на заболеваемость, проведенные на больших статистических выборках случаев (до нескольких миллионов) с ис- пользованием многопараметрических подходов, показали относительность, нелинейность, немонотонность метеоэффекта, существование значительного временного лага в его развитии, а также зависимость степени его проявления от сезона года и от географической локализации места проживания.

Деление выборки пациентов на группы по разным признакам показало, что выраженность метеоэффекта зависит от возраста и пола человека, а также расы, питания, социального статуса, образа жизни (активный или малоподвижный).

Большинство авторов приходит к заключению, что наиболее биотропным фактором является приземная температура воздуха, ее текущие значения и амплитуда вариаций. Однако ежедневные вариации разных метеопараметров сильно скоррелированы, что необходимо учитывать при анализе.

Изучать характеристики метеоэффекта по отношению к «сердечно-сосудистым заболеваниям в целом» методологически неправомерно, поскольку кривые риска развития различных заболеваний в зависимости от метеофакторов имеют кардинально отличающиеся профили.

Можно экспериментально выделить области значений метеопараметров, в которых существенно возрастает чувствительность организма к действию других внешних факторов, например геомагнитной активности.

В развитии метеотропной реакции организма задействованы биохимические показатели крови, внезапное изменение которых может служить как индикатором метеотропной реакции пациента, так и непосредственной причиной резкого ухудшения самочувствия или даже смерти.

Результаты, полученные в разных исследованиях, значительно различаются по особенностям и даже знаку эффекта, величине временного лага развития реакции и ее амплитуде.

При сопоставлении результатов близких по дизайну исследований, проведенных разными научными группами, всегда существует ряд неучтенных причин, которыми можно предположительно объяснить расхождение полученных результатов, если оно обнаруживается. В то же время экспериментально проверить справедливость данных предположений крайне сложно.

Сегодня, учитывая перечисленные выше уже установленные свойства метеоэффекта, а также его вариабельность по нескольким классам параметров, можно сделать вывод, что конечный результат исследования в значительной степени зависит от параметров усреднения эффекта, выбранных его авторами. Так, если с ростом температуры риск развития инфарктов возрастает, а инсультов - снижается, то объединение в исследовании этих двух заболеваний в усредненный класс «сердечно-сосудистых осложнений» может привести к отсутствию зависимости. 
Однако существует еще один принципиально важный аспект проблемы метеочувствительности, а именно значительная межиндивидуальная вариабельность. Суть ее в том, что отдельные участники экспериментальной группы, однородной по всем известным критериям включения, на действие одного и того же метеофактора могут реагировать противоположным образом, например часть пациентов ростом, а другая часть - снижением показателей АД.

О возможном пути преодоления данной проблемы пойдет речь во второй части данной работы.

\section{2. Индивидуальный мониторинг физиологических параметров}

\section{1. История вопроса}

В 1992 г. российские физиологи В.Н. Шеповальников и С.И. Сороко [Шеповальников, Сороко, 1992] описали результаты длительного наблюдения группы полярников в условиях арктических и антарктических зимовок. По-видимому, это было одно из первых исследований, в котором удалось получить и проанализировать длинные временные ряды индивидуальных измерений параметров АД и ЧСС большой, достаточно однородной группы здоровых мужчин среднего возраста.

Результаты, полученные ими на основе одних и тех же данных при общегрупповом и при индивидуальном анализе, показали, что операция усреднения по группе скрывает очень важные особенности эффекта, а иногда и сам эффект.

В наших работах начиная с 2009 г. было показано, что синтез всего многообразия возможных индивидуальных реакций и построение на этой основе общей картины метеоэффекта возможны, если изменить и дополнить традиционный алгоритм анализа данных. Предлагаемый нами новый подход, показавший свою эффективность, состоит из нескольких этапов:

- получение у максимально широкого круга лиц длинных временных рядов значений физиологических показателей, таких как АД и ЧСС, показателей вариабельности сердечного ритма, позволяющих оценить степень активации различных отделов вегетативной нервной системы;

- расчет для каждого человека отдельно численных характеристик его персональной метеотропной реакции на каждый из метеофакторов, таких как амплитуда реакции, ее длительность, временной лаг;

- сохранение полученных численных характеристик в единой базе знаний вместе со входными параметрами: пол, возраст, рост, вес, хронические заболевания, время, место и средние значения метеопараметров проведения наблюдений;

- анализ не усредненного по группе значения амплитуды метеореакции с определенными процентиля- ми, а самого распределения полученных выборочных значений при каждом наборе входных параметров;

- анализ изменения формы и характеристик этого распределения при изменении всех по очереди входных параметров.

Использование этого алгоритма позволяет не предполагать а-приори, а определить экспериментально, от каких из входных параметров действительно зависит выраженность метеоэффекта, отсортировать их по важности, сформулировать критерии включения и исключения при клинических исследованиях.

\section{2. Анализ результатов, полученных на основе индивидуального подхода к оценке \\ метеочувствительности и проблема поиска наиболее биотропного фактора}

Ответ на вопрос о том, какой из атмосферных факторов оказывает наиболее сильное влияние, разными исследователями решался и до сих пор решается по-разному.

Ряд авторов наиболее биотропным фактором признает атмосферное давление, направление его изменения или интенсивность его высокочастотных вариаций (микробаромов).

Так, в работе [Delyukov et al., 2001] продемонстрирована статистическая зависимость основных показателей вариабельности сердечного ритма здорового волонтера от уровня геомагнитной активности (ГМА), температуры воздуха, относительной влажности и силы ветра. Но наиболее тесная зависимость была обнаружена с интенсивностью флуктуаций атмосферного давления в интервале от 0,01 до 0,1 Гц (микробаромами).

В работе [Ozheredov et al., 2017] доказано существование определенных областей значений атмосферных факторов, при которых отклик организма на геомагнитные возмущения проявляется наиболее выраженно. Материалами для анализа здесь послужили результаты 7-дневных записей показателей АД и ЧСС у 197 здоровых волонтеров в возрасте от 18 до 27 лет, проживающих в Москве.

Атмосферное давление в этом исследовании, также как и в [Ожередов и др., 2010], оказывается основным фактором влияния и обеспечивает магнитобиотропность для всех рассмотренных физиологических параметров. Из предыдущих работ известно, что основное влияние геомагнитные факторы оказывают на тонус сосудов, как крупных, так и мелких, и специфическая реакция на ГМА аналогична реакции на метеорологические факторы [Зенченко и др., 2009; Бреус и др., 2010; Ожередов и др., 2010; Gurfinkel et al., 2012].

К сожалению, указанные работы являются уникальными в литературе по постановке задачи и ме- 
тодам анализа, поэтому сравнить их с близкими по дизайну исследованиями невозможно.

Северные иироты. Цикл исследований, объединенный общим подходом к сбору и анализу данных, примененный к результатам наблюдений здоровых жителей разных широт, показал, что для Севера России и средних широт таким наиболее биотропным фактором является температура воздуха.

Так, в работах [Зенченко, Варламова, 2015; Варламова и др., 2017] на основе как индивидуального, так и среднегруппового анализа делается вывод, что вариации температуры воздуха являются наиболее биотропным фактором, влияющим на изменение АД и ЧСС здоровых волонтеров, жителей северных широт. В данном случае влияют как сезонные вариации, так и волны длительностью в несколько суток.

В результатах, полученных в [Зенченко, Варламова, 2015], можно выделить три отчетливо различающихся уровня зависимости гемодинамических показателей от внешних факторов (рис. 2). Самый высокий уровень (70-80 \% случаев) характерен для показателей сиастолического (САД) и диастолического (ДАД) с $\mathrm{T}_{\text {атм }}$ и функционально связанными с ним парциальным содержанием кислорода в воздухе $\mathrm{P}\left(\mathrm{O}_{2}\right)$. Второй уровень - 30-50\% характерен для за-

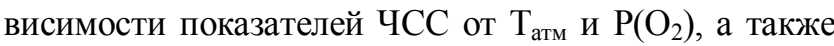
всех трех гемодинамических показателей от уровня геомагнитной активности, выражаемой Кр-индексом. К третьему уровню, практически означающему отсутствие статистической связи, можно отнести зависимость САД, ДАД и ЧСС от $\mathrm{P}_{\text {атм }}, \mathrm{dP}$ и $\mathrm{dT}$.

Важно отметить, что в период наблюдений усредненные по сезонам значения атмосферных показателей находились в границах многолетней нормы для данного региона. В то же время уровень ГМА был близок к максимуму данного солнечного цикла.

Вывод о доминирующей роли вариаций температуры в изменениях показателей АД и вариабельности сердечного ритма у здоровых мужчин молодого и среднего возраста в условиях Севера сделан также в работе [Markov et al., 2015]. Во всех проведенных нами исследованиях здоровых волонтеров Севера, мужчин и женщин разного возраста [Зенченко и др., 2011; Markov et al., 2015, Зенченко, Варламова, 2015] во всех статистически значимых случаях знак корреляции между изменениями АД и $\mathrm{T}_{\text {атм }}$ был отрицательным (от 60 до 85 \% волонтеров каждой группы).

Средние ииротьл. Аналогичные исследования метеочувствительности показателей АД и ЧСС, а также показателей микроциркуляции крови в периферических сосудах [Зенченко и др., 2009, 2013], проведенные по аналогичной методике, но для жителей средних широт (г. Москвы) в разные годы наблюдений, дали аналогичный вывод.
Оказалось, что для климатического пояса Москвы наиболее биотропным для здоровых людей атмосферным фактором являются вариации температуры воздуха. Понижение температуры приводило к повышению как тонуса магистральных сосудов (рост АД), так и мелких сосудов субкапиллярного плетения, и, как следствие, к снижению показателей микроциркуляции крови в мелких сосудах.

Однако знак реакции на температуру в средних широтах, в отличие от Севера, варьировал в разных исследованиях. Например, в работе [Зенченко и др., 2011] описана ситуация, когда в двух группах волонтеров г. Москвы, однородных по всем параметрам, кроме года наблюдения, были получены противоположные преобладающие знаки корреляции между АД и $\mathrm{T}_{\text {атм. }}$

Так, при разбиении более чем трехлетнего ежедневного ряда измерений АД и ЧСС здорового волонтера на 14 неперекрывающихся отрезков, соответствующих календарным сезонам года, было получено, что для 11 из 14 временных интервалов существует статистически значимая $(p<0,05)$, всегда отрицательная, корреляция показателей АД с $\mathrm{T}_{\text {атм }}$ [Зенченко и др., 2013].

Однако в другом длительном эксперименте (рис. 3) было обнаружено, что у одного и того же волонтера (мужчина, 67 лет, житель г. Москва) три года подряд в течение летних месяцев с умеренными среднесезонными температурами (2007-2009 гг.) корреляции показателей САД с $\mathrm{T}_{\text {атм }}$ были отрицательными, однако в течение экстремально жаркого лета 2010 г. знак корреляции поменялся на противоположный.

Южные широты. Качественно по-другому выглядит картина реакции здоровых людей на метеофакторы в условиях южных широт (г. Симферополь). Здесь проводился целый ряд исследований [Зенченко и др., 2008; Поскотинова, Григорьев, 2008] результаты которых показывают, что наибольший процент случаев зависимости наблюдается от параметра ГМА, а из атмосферных факторов - от атмосферного давления. При этом внутри каждой из групп наблюдались оба знака реакции на $\mathrm{T}_{\text {атм. }}$.

Таким образом, если обобщить результаты индивидуального анализа здоровых волонтеров различных широт, проведенного по рядам наблюдений длиной порядка 100 точек каждый, получаем, что:

- в высоких и средних широтах доминирующим фактором, влияющим на 60-90 \% населения, оказывается температура воздуха; в южных широтах атмосферное давление;

- знак корреляции с $\mathrm{T}_{\text {атм }}$ в северных широтах наблюдался всегда отрицательный, в средних и южных широтах встречались оба знака. 


\section{3. Выявление типов реакциии}

Эту разнонаправленность наблюдаемых реакций удалось объяснить [Зенченко и др., 2013] в результате анализа формы зависимости показателей АД от $\mathrm{T}_{\text {атм }}$ в широком диапазоне температур на основании многолетних рядов индивидуальных наблюдений. Оказа- лось, что эта зависимость является нелинейной и в некоторых случаях немонотонной, однако воспроизводится в основных чертах у разных волонтеров.

На основании многолетних ежедневных наблюдений семи волонтеров были выделены два отчетливых типа реакции показателей АД и ЧСС на изменение $\mathrm{T}_{\text {атм }}$ (рис. 4).

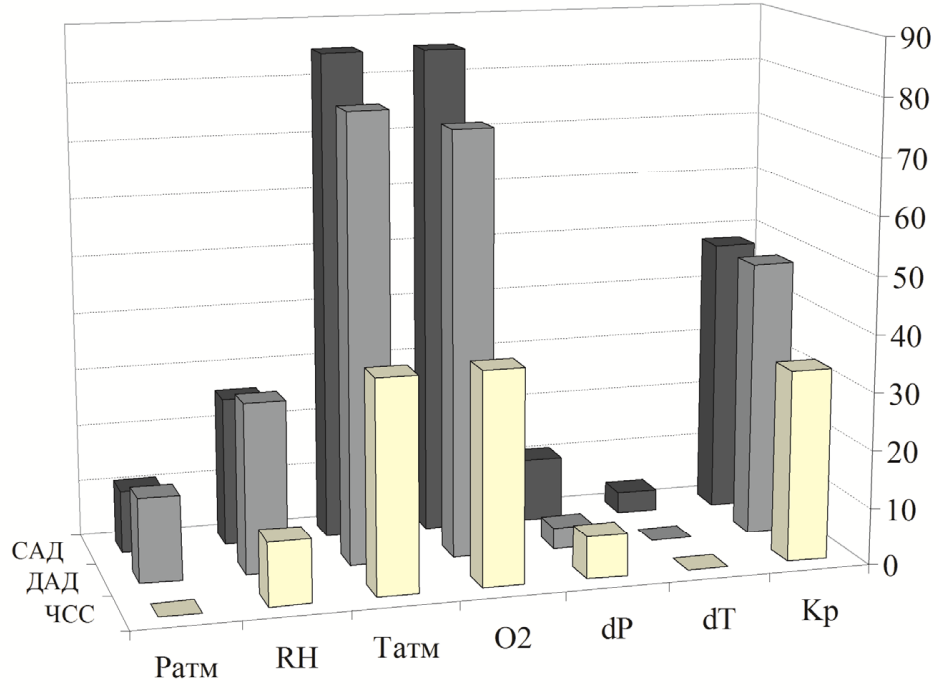

Рис. 2. Распределение случаев значимой корреляции физиологических показателей с метеорологическими и геомагнитными параметрами для группы волонтеров Сыктывкара

По оси абсцисс перечислены параметры окружающей среды, по оси ординат - исследованные физиологические показатели. Высота столбика по оси аппликат указывает, у какого процента волонтеров наблюдалась значимая $(p<0,05)$ непараметрическая корреляция между данными показателями

Fig. 2. Percentage of significant correlation cases of BP with meteorological and geomagnetic parameters for a group of Syktyvkar volunteers (27 people)

The bar height along $\mathrm{Z}$ - direction indicates the percentage of volunteers that showed a significant $(p<0,05)$ nonparametric correlation between these indicators
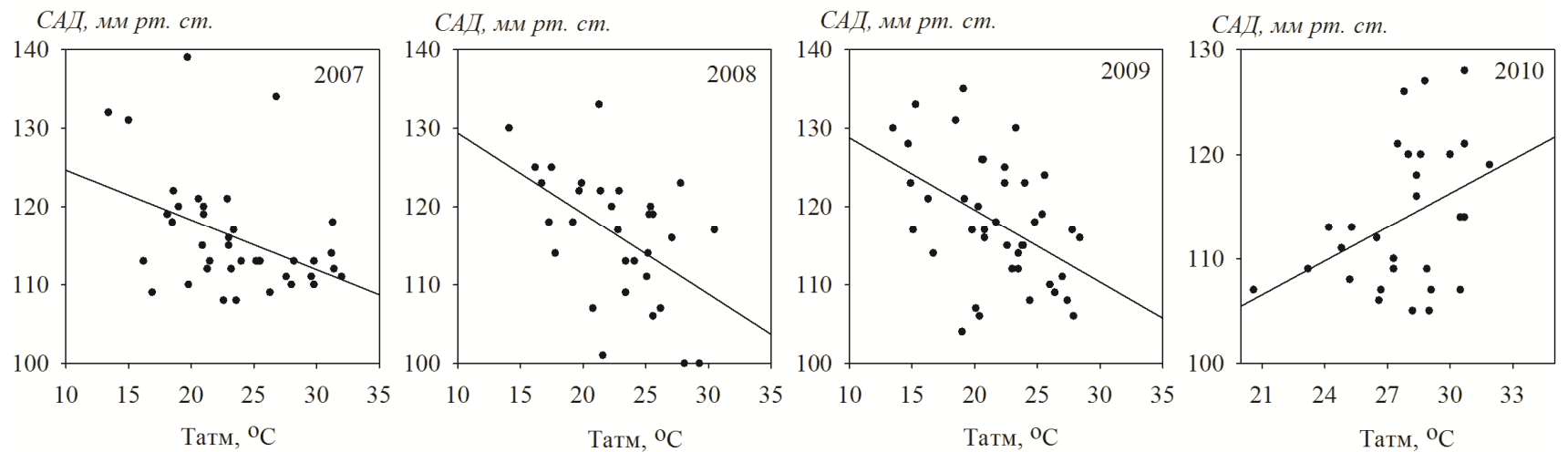

Рис. 3. Примеры зависимости значений САД от температуры воздуха, измеренные у одного волонтера (мужчина, 67 лет, житель г. Москва) в течение четырех последовательных летних сезонов Все представленные зависимости статистически значимы $(p<0,05)$

Fig. 3. Examples of distributions of SBP values from outdoor temperature, measured in one volunteer (male, 67 years old, resident of Moscow) for four consecutive summer seasons

All presented dependences are statistically significant $(p<0,05)$ 
$a$

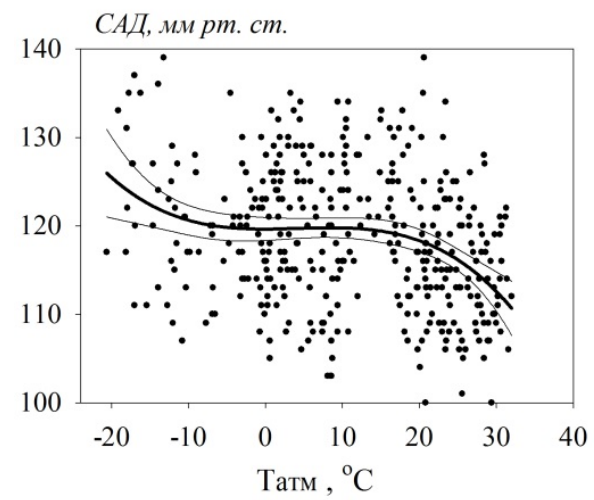

$b$

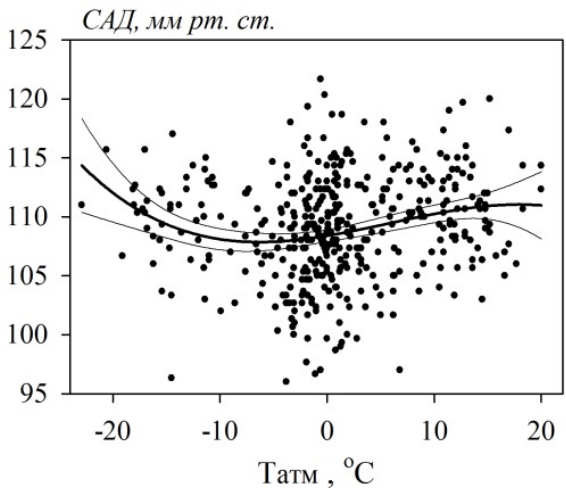

Рис. 4. Примеры типичных зависимостей первого (a) и второго (b) типов реакции показателей САД от значений $\mathrm{T}_{\text {атм }}$

Приведены линии регрессии полиномом третьего порядка (с 95 \%-ми доверительными интервалами)

Fig. 4. Examples of the first ( $a$ ) and second $(b)$ types of systolic $B P$ reaction on $T_{\text {atm }}$ Regression lines are shown as a third-order polynomial (with $95 \%$ confidence intervals)

Первый тип (рис. $4, a)$ - снижение уровня систолического (и в меньшей степени - диастолического) АД по мере роста температуры воздуха, монотонное, но неравномерное по скорости. Наиболее отчетливо снижение АД выражено на концах интервала, при $25^{\circ} \mathrm{C}<\mathrm{T}_{\text {атм }}<-5{ }^{\circ} \mathrm{C}$ и $15^{\circ} \mathrm{C}<\mathrm{T}_{\text {атм }}<32{ }^{\circ} \mathrm{C}$, а при средних температурах зависимость почти отсутствует.

Второй тип реакции (рис. $4, b$ ) - немонотонная двухфазная зависимость показателей АД от $\mathrm{T}_{\text {атм. }}$ В диапазоне $\mathrm{T}_{\text {атм }}<-5^{\circ} \mathrm{C}$ она совпадает с первым типом (чем ниже температура, тем выше АД), а при $-5{ }^{\circ} \mathrm{C}<\mathrm{T}_{\text {атм }}<32{ }^{\circ} \mathrm{C}$ характеризуется положительной корреляцией показателей САД, ДАД и ЧСС с $\mathrm{T}_{\text {атм. }}$.

Если при отрицательных температурах с усилением интенсивности морозов всегда наблюдался рост АД, то при положительных температурах для одних волонтеров была характерна положительная корреляция, для других - отрицательная. Интересно отметить, что для обоих типов реакции у всех волонтеров, для которых были получены многолетние ряды наблюдений, точкой смены режима реакции оказалась температура, близкая к $-5^{\circ} \mathrm{C}$.

Понимание того факта, что знак реакции может быть различным не только у разных людей, но и одного человека в разных диапазонах температур, позволило объяснить разнонаправленность результатов индивидуального анализа, полученных в предыдущих работах на более коротких отрезках наблюдений.

Обнаружено, что у волонтеров в работах [Gurfinkel et al., 2012; Зенченко, Варламова, 2015] наблюдался первый тип зависимости, аналогичный рис. $4, a$.

Учет температурного диапазона в период наблюдений позволил объяснить разнонаправленность ре- акций АД на температуру воздуха у 17 молодых волонтеров г. Симферополя [Зенченко и др., 2008], у 33 пациентов в диагнозом артериальная гипертензия 1-й и 2-й степени, жителей Москвы, а также объяснить наблюдаемые у некоторых из них «ускользания гипотензивного эффекта» [Зенченко и др., 2007]. Важно подчеркнуть, что во всех этих случаях измерений положительные коэффициенты корреляции наблюдали либо при нулевых, либо при положительных температурах, так что все полученные ра-

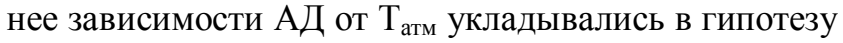
о двух типах реакции, в том числе и по границам температурных диапазонов.

Таким образом, результаты, полученные на основании цикла из десяти работ, указывают на то, что реакция показателей АД на изменение температуры воздуха зависит в первую очередь не от места проживания человека, а от текущих значений параметров погоды.

Для точного ответа на вопрос о зависимости характеристик метеотропного эффекта от возраста и пола человека пока не достаточно собранных данных. В настоящий момент изучена реакция всего 390 человек, в возрасте от 18 до 67 лет. Можно только утверждать, что эффекту метеочувствительности подвержены как мужчины, так и женщины, и что по показателю доли метеочувствительных людей не обнаружено возрастных исключений или преимуществ ни в одной из групп.

\section{4. Возможные механизмы реакциии}

В обзорах, посвященных действию экстремальных температур, перечислены возможные механиз- 
мы развития физиологической реакции, приводящей в итоге к сердечно-сосудистым катастрофам именно в результате перегрева организма [Kenney et al., 2014].

В то же время многочисленные работы, процитированные выше, говорят о существовании реакции организма на вариации наружной температуры при значениях, далеких от экстремальных. Это утверждение вызывает скепсис и возражение ряда исследователей, поскольку обычно измерение показателей АД происходит в помещении, а изменения наружной и комнатной температуры коррелируют слабо. В этой ситуации появляется необходимость поиска механизмов реакции организма, отличных от прямого перегрева или переохлаждения.

Использование индивидуального подхода позволило не только доказать широко распространенную метеочувствительность здоровых лиц и выделить типы реакции, но и построить подробную схему возможных последовательных физиологических процессов, развивающихся в организме при определенных диапазонах температуры воздуха и приводящих к наблюдаемым сдвигам АД [Зенченко и др., 2013]. Причем, если возможные физиологические и биохимические механизмы длительного воздействия экстремально низких значений температуры воздуха на организм здоровых людей подробно исследованы на протяжении многих лет [см., например, обзор Patrakeeva, Basova, 2018], то диапазон умеренных температур и возможной реакции на него показателей АД ранее не исследовался.

Первый тип реакции (рис. 4, a) скорее всего, обусловлен сочетанием разных физиологических механизмов, обеспечивающих развитие компенсаторноприспособительной реакции в разных температурных диапазонах. Так, при $15{ }^{\circ} \mathrm{C}<\mathrm{T}_{\text {атм }}<32{ }^{\circ} \mathrm{C}$ реакция организма может быть вызвана активацией центральных гипоталамических структур, снижением тонуса вен и артерий кожи и снижением объема циркулирующей жидкости.

При $-25^{\circ} \mathrm{C}<\mathrm{T}_{\text {атм }}<-5^{\circ} \mathrm{C}$, для обоих типов реакции феноменологическая картина совпадает, и, повидимому, в этой области доминирует эффект, связанный с активацией работы щитовидной железы при усилении морозов.

Второй тип реакции (рис. 4, $b$ ) при $-5^{\circ} \mathrm{C}<\mathrm{T}_{\text {атм }}<32{ }^{\circ} \mathrm{C}$ по всему комплексу признаков совпадает с развитием компенсаторно-приспособительной реакции на недостаток содержания кислорода в воздухе (внешняя гипоксия). Зависимость от парциального содержания кислорода наблюдается именно в области пониженных значений $\mathrm{P}\left(\mathrm{O}_{2}\right)$.

Существует только два атмосферных параметра, значения которых тесно скоррелированы в помещении и на улице: атмосферное давление и парциальное содержание кислорода в воздухе. Однако, как видно из приведенных в данной работе примеров, зависимость от $\mathrm{P}_{\text {атм }}$ обнаруживается в относительно небольшом проценте исследований.

Возможная роль вариаций атмосферного кислорода как непосредственно действующего фактора обсуждалась начиная с 1980-х гг. [Овчарова, 1981]. Парциальное содержание кислорода в воздухе жестко связано со значениями температуры при $\mathrm{T}_{\text {атм }}>0$, поэтому при положительных умеренных температурах действие Татм может быть обусловлено снижением концентрации кислорода.

Подтверждение биотропной роли парциального содержания кислорода было получено в работе [Кузнецова и др., 2019]. У лабораторных животных контрольной группы, никогда не контактировавших с атмосферой вне помещения, в эксперименте периодически наблюдались многократно повышенные значения биохимических показателей. Было получено, что все такие значения располагались в окрестности нуля температуры и парциального содержания кислорода воздуха 298-304 мг/л, т.е. в диапазоне повышенной генерации активных форм атмосферного кислорода.

Таким образом, в работе было получено подтверждение двух важных аспектов проблемы метеочувствительности человека к изменениям именно приземной температуры воздуха. Во-первых, было доказано существование данного эффекта для лабораторных животных, никогда не контактировавших напрямую с уличной температурой. Во-вторых, в результате удачного выбора изучаемых биологических параметров, чувствительных к процессам окисления, удалось показать, что значимую роль в механизме развития данного типа метеореакции играет именно атмосферный кислород.

\section{Заключение}

Популяционные исследования, проведенные на больших статистических выборках случаев заболеваемости, показали доминирующую роль приземной температуры воздуха как в области экстремальных, так и в области фоновых значений. Также была показана относительность, нелинейность, немонотонность метеоэффекта, существование значительного временного лага в его развитии, а также зависимость степени его проявления от сезона года и географической локализации места проживания.

Выраженность популяционного метеоэффекта зависит от возраста и пола человека, возможно, расы, питания, социального статуса, образа жизни (активный или малоподвижный), а также от конкретной сопутствующей нозологии. Практически любые заболевания, характерные для людей старшего возраста, увеличивают риск смерти при наступлении волн жары или холода. 
Биохимические показатели в популяционных исследованиях, такие как сердечно-сосудистые маркеры и липопротеины, не только показывают чувствительность к изменениям температуры воздуха, но и позволяют построить возможную модель развития реакции организма на уровне физиологии. Внезапное изменение этих показателей может служить индикатором метеотропной реакции пациента и непосредственной причиной резкого ухудшения самочувствия или даже смерти.

В то же время популяционный подход обладает рядом методологических ограничений, которые можно в значительной степени преодолеть в рамках добавления к нему индивидуального подхода.

В первую очередь, это выявление метеоэффекта в тех случаях, где он оказывается замаскированным в результате применения операции усреднения по группе, времени наблюдения или по температурному диапазону. Например, как было показано в рамках индивидуального подхода, в северных широтах направление изменения показателей АД под действием вариаций температуры воздуха практически всегда одинаково, в южных - разнонаправленное. При условии применения исключительно популяционного подхода при некоторых условиях может быть сделан вывод об отсутствии метеоэффекта в низких широтах в диапазоне умеренных температур. Аналогичная маскировка эффекта может происходить при выборе слишком широкого диапазона изменения температур, внутри которого зависимость немонотонна. Учет особенностей метеоэффекта, полученных в рамках индивидуального подхода, позволяет избежать потери информации.

Во-вторых, индивидуальный подход может помочь в решении проблемы объяснения конкретных причин вариабельности эффекта. Очень часто при сходстве входных условий популяционного анализа в разных исследованиях получаются различные результаты. Обычно существует перечень факторов, физических, медико-анамнестических или математических, которые потенциально могут быть причиной таких различий, однако для точного объяснения информации бывает недостаточно. В этой ситуации наличие обширной базы результатов индивидуального анализа позволяет не предполагать априори, а определить экспериментально, от каких из входных параметров, помимо общепринятных (пола, возраста и т.д.), действительно зависит выраженность метеоэффекта, отсортировать их по важности, сформулировать критерии включения и исключения при клинических исследованиях. Как было показано в данной работе, в частности, такими факторами являются географическая широта места наблюдения и текущие значения температуры воздуха.

Однако использование только индивидуального подхода малоэффективно, поскольку в настоящее время он не позволяет достичь необходимого популяционного охвата и получить столь обширные базы данных и столь статистически надежные выводы, как популяционный подход.

Таким образом, расширение методов исследования метеоэффекта за счет включения метода индивидуального анализа позволяет значительно увеличить степень детализации информации об особенностях метеоэффекта, преодолеть ряд ограничений, свойственных популяционному подходу, и таким образом дополнить общую картину, получаемую в массовых популяционных исследованиях заболеваемости, обусловленной метеофакторами.

\section{ЛИТЕРАТУРА}

Андронова Т.И., Деряпа Н.Р., Соломатин А.П. Гелиометеотропные реакции здорового и больного человека. Л. : Медицина, 1982. $247 \mathrm{c}$.

Ассман Д. Чувствительность человека к погоде. Л. : Гидрометеоиздат, 1966. 245 с.

Бреус Т.К., Гурфинкель Ю.И., Зенченко Т.А., Ожередов В.А. Сравнительный анализ чувствительности различных показателей сосудистого тонуса к метеорологическим и геомагнитным факторам // Геофизические процессы и биосфера. 2010. Т. 9 , № 2. C. 23-36. DOI: 10.1134/S0001433810080050

Бреус Т.К., Бинги В.Н., Петрукович А.А. Магнитный фактор солнечно-земных связей и его влияние на человека: физические проблемы и перспективы // Успехи физических наук. 2016. Т. 186, № 5. С. 568-576.

Варламова Н.Г., Зенченко Т.А., Бойко Е.Р. Годовая динамика артериального давления и метеочувствительность у женщин // Терапевтический архив. 2017. Т. 12. С. 56-63. DOI: 10.17116/terarkh2017891256-63

Зенченко Т.А., Цагареишвили Е.В., Ощепкова Е.В., Рогоза А.Н., Бреус Т.К. К вопросам влияния геомагнитной и метеорологической активности на больных артериальной гипертонией // Клиническая медицина. 2007. № 1. С. 31-35.

Зенченко Т.А., Цандеков П.А., Григорьев П.Е., Мёрзлый А.М., Зенченко К.И., Хорсева Н.И., Григал П.П. Исследование характера связей физиологических и психофизиологических показателей организма с метеорологическими и геомагнитными факторами // Геофизические процессы и биосфера. 2008. № 3. С. 25-36.

Зенченко Т.А., Рехтина А.Г., Хорсева Н.И., Заславская Р.М., Бреус Т.К. Сравнительный анализ чувствительности различных показателей гемодинамики здоровых людей к действию атмосферных факторов в условиях средних широт // Геофизические процессы и биосфера. 2009. Т. 8, № 4. С. 61-76.

Зенченко Т.А., Мёрзлый А.М., Солонин Ю.Г. Сравнение случаев индивидуальной метеочувствительности человека в экстремальных условиях зимы северных и средних широт // Экология человека. 2011. № 11. С. 3-13.

Зенченко Т.А. Метод последовательных приближений в задаче исследования механизма индивидуальных гелиометеотропных реакций // Сборник материалов международной конференции «Влияние космической погоды на здоровье человека в космосе и на Земле». М., 2012. С. 633-648. 
Зенченко Т.А., Скавуляк А.Н., Хорсева Н.И., Бреус Т.К. Характеристики индивидуальных реакций сердечно-сосудистой системы здоровых людей на изменение метеорологических факторов в широком диапазоне температур // Геофизические процессы и биосфера. 2013. № 1. С. 22-43.

Зенченко Т.А., Варламова Н.Г. Характеристики реакции показателей гемодинамики здоровых людей на изменения метеорологических и геомагнитных факторов в условиях Севера // Геофизические процессы и биосфера. 2015. Т. 14, № 2. С. 50-66.

Кузнецова Е.А., Сирота Н.П., Зенченко Т.А. Изменение уровней повреждений ДНК клеток мышей в зависимости от атмосферных факторов // Биофизика. 2019. Т. 64, № 3. С. 457-467. DOI: 10.1134/S0006302919030050

Овчарова В.Ф. Определение содержания кислорода в атмосферном воздухе на основе метеорологических параметров (давления, температуры, влажности) с целью прогнозирования гипоксического эффекта атмосферы // Вопросы курортологии, физиотерапии и лечебной физкультуры. 1981. № 2. С. 29-34.

Ожередов В.А., Бреус Т.К., Гурфинкель Ю.И., Ревич Б.А, Митрофанова Т.А. Влияние космической и земной погоды на развитие острых кардиологических патологий // Биофизика. 2010. Т. 55, № 1. С. 133-144.

Поскотинова Л.В., Григорьев П.Е. Зависимость типологических особенностей вегетативных реакций здоровых лиц от фоновых показателей // Экология человека. 2008. № 5. С. 3-8.

Шеповальников В.Н., Сороко С.И. Метеочувствительность человека / отв. ред. В.А. Яковлев. Бишкек : Илим, 1992.247 с.

Alperovitch A., Lacombe J.M., Hanon O., Dartigues J.F., Ritchie K., Ducimetière P., Tzourio C. Relationship between blood pressure and outdoor temperature in a large sample of elderly individuals: The Three-City study // Arch. Intern. Med. 2009. 169. P. 7580. DOI: $10.1001 /$ archinternmed.2008.512

Armstrong B. Models for the relationship between ambient temperature and daily mortality // Epidemiology. 2006. No 17 (6). P. 624-631. DOI: 10.1097/01.ede.0000239732.50999.8f

Azcárate T., Mendoza B. Influence of geomagnetic activity and atmospheric pressure in hypertensive adults // Int. J. Biometeorol. 2017. No 61. P. 1585-1592. DOI: 10.1007/s00484-017-1337-x

Basu R. High ambient temperature and mortality: a review of epidemiologic studies from 2001 to 2008 // Environ Health. 2009. No 8. P. 40.

Basu R., Pearson D., Malig B., Broadwin R., Green R. The effect of high ambient temperature on emergency room visits // Epidemiology. 2012. No 23. P. 813-820. DOI: 10.1097/EDE.0b013e31826b7f97.

Bayentin L., El Adlouni S., Ouarda T.B., Gosselin P., Doyon B., Chebana F. Spatial variability of climate effects on ischemic heart disease hospitalization rates for the period 1989-2006 in Quebec, Canada // Int. J. Health Geogr. 2010. No 9. P. 5. DOI: 10.1186/1476-072X-9-5

Berginer V.M., Goldsmith J., Batz U., Vardi H., Shapiro Y. Clustering of strokes in association with meteorologic factors in the Negev desert of Israel: 1981-1983// Stroke. 1989. No 20. P. 65-69. DOI: 10.1161/01.STR.20.1.65

Bhaskaran K., Hajat S., Haines A., Herrett E., Wilkinson P., Smeeth L. Effects of ambient temperature on the incidence of myocardial infarction // Heart. 2009. No 95. P. 1760-1769. DOI: 10.1136/hrt.2009.175000

Casas A.L.F., Santos G.M.D., Chiocheti N.B., de Andrade M. Effects of Temperature Variation on the Human Cardiovascular System: A Systematic Review // Climate Change and Health. Climate Change Management. Cham, Switzerland : Springer, 2016.

Comelli I., Ferro J., Lippi G., Comelli D., Sartori E., Cervellin G. Incidence of acute-onset atrial fibrillation correlates with air temperature. Results of a nine-year survey // J. Epidemiol. Glob. Health. 2014. No 4. P. 151-157. DOI: 10.1016/j.jegh.2013.12.003

Čulič V., Eterovič D., Mirič D., Giunio L., Lukin A., Fabijanič D. Triggering of ventricular tachycardia by meteorologic and emotional stress: protective effect of b-blockers and anxiolytics in men and elderly // Am. J. Epidemiol. 2004. No 160. P. $1047-1058$. DOI: 10.1093/aje/kwh335

Danet S., Richard F., Montaye M., Beauchant S., Lemaire B., Graux C., Cottel D., Marécaux N., Amouyel P. Unhealthy effects of atmospheric temperature and pressure on the occurrence of myocardial infarction and coronary deaths. A 10-Year Survey: The Lille-World Health Organization MONICA Project (Monitoring trends and determinants in cardiovascular disease) // Circulation. 1999. No 100. e1-e7. DOI: 10.1161/01.CIR.100.1.e1

Davis R.E., Hondula D.M., Sharif H. Examining the diurnal temperature range enigma: why is human health related to the daily change in temperature? // Int. J. Biometeorol. 2020. No 64. P. 397-407. DOI: 10.1007/s00484-019-01825-8

Delyukov A., Gorgo Y., Cornélissen G. et al. Natural environmental associations in a 50-day human electrocardiogram // Int. J. Biometeorol. 2001. No 45 (2). P. 90-99. DOI: 10.1007/s004840100086

Dockery D.W., Pope C.A. 3rd, Kanner R.E., Villegas M.G., Schwartz J. Daily changes in oxygen saturation and pulse rate associated with particulate air pollution and barometric pressure // Res. Rep. Health Eff. Inst. 1999. No 83. P. 1-19. PMID: 10192116

Ebi K.L., Exuzides K.A., Lau E., Kelsh M., Barnston A. Weather changes associated with hospitalizations for cardiovascular diseases and stroke in California, 1983-1998 // Int. J. Biometeorol. 2004. No 49. P. 48-58. DOI: 10.1007/s00484-004-0207-5

Feigin V.L., Nikitin Y.P., Bots M.L., Vinogradova T.E., Grobbee D.E. A population-based study of the associations of stroke occurrence with weather parameters in Siberia, Russia (1982-92) // Eur. J. Neurol. 2000. No 7. P. 171-178. DOI: 10.1046/j.14681331.2000.00016.x

Głuszak A., Kocoń S., Zuk K., Aljabali P., Gluza A., Siwek K. Episodes of atrial fibrillation and meteorological conditions // Kardiol Pol. 2008. No 66. P. 958-963.

Gurfinkel Y.I., Breus T.K., Zenchenko T.A., Ozheredov V.A. Investigation of the effect of ambient temperature and geomagnetic activity on the vascular parameters of healthy volunteers // Open J. Biophys. 2012. No 2. P. 46-55. DOI: 10.4236/ojbiphy.2012.22007

Halonen J.I., Zanobetti A., Sparrow D., Vokonas P.S., Schwartz J. Relationship between outdoor temperature and blood pressure // Occup. Environ. Med. 2011. No 68. P. 296-301. DOI: 10.1136/oem.2010.056507

Hong Y.C., Kim H., Oh S.Y., Lim Y.H., Kim S.Y., Yoon H.J. et al Association of cold ambient temperature and cardiovascular markers // Sci. Total Environ. 2012. No 435-436. P. 74-79. DOI: 10.1016/j.scitotenv.2012.02.070

Houck P., Lethen J., Riggs M., Gantt S., Dehmer G. Relation of atmospheric pressure changes and the occurrences of acute myocardial infarction and stroke // Am. J. Cardiol. 2005. No 96. P 45-51. DOI: 10.1016/j.amjcard.2005.02.042

Jie Y., Houjin H., Mengxue Y., Wei Q., Jie X. A time series analysis of meteorological factors and hospital outpatient admissions for cardiovascular disease in the Northern district of Guizhou Province, China // Braz. J. Med. Biol. Res. 2014. No 47 (8). P. $689-696$. DOI: $10.1590 / 1414-431 X 2014424$ 
Kenney W.L., Craighead D.H., Alexander L.M. Heat waves, aging, and human cardiovascular health // Med. Sci Sports Exerc. 2014. No 46 (10). P. 1891-1899. DOI: 10.1249/MSS.0000000000000325

Kulakov I.V., Nasonova E.V. The specific features of circadian blood pressure variations in patients with hypertensive disease in different types of weather// Klin. Med. 2004. No 82. P. 24-27. PMID: 15468719

Lavigne E., Gasparrini A., Wang X., Yagouti A., Fleury M.D., Cakmak S. Extreme ambient temperatures and cardiorespiratory emergency room visits: assessing risk by comorbid health conditions in a time series study // Environ Health. 2014. No 13. P. 5. DOI: 10.1186/1476-069X-13-5

Lin S., Luo M., Walker R.J., Liu X., Hwang S.A., Chinery R. Extreme high temperatures and hospital admissions for respiratory and cardiovascular diseases // Epidemiology. 2009. No 20. P. 738-746. DOI: 10.1097/EDE.0b013e3181ad5522

Linares C., Díaz J. Impact of high temperatures on hospital admissions: comparative analysis with previous studies about mortality (Madrid) // The European Journal of Public Health. 2008. No 18 (3). P. 317-322. DOI: 10.1093/eurpub/ckm108

Madaniyazi L., Guo Y., Williams G. et al. The nonlinear association between outdoor temperature and cholesterol levels, with modifying effect of individual characteristics and behaviors // Int. J. Biometeorol. 2020. No 64. P. 367-375. DOI: 10.1007/s00484-01901816-9

Magalhães R., Silva M.C., Corrêia M., Bailey T. Are stroke occurrence and outcome related to weather parameters? Results from a population-based study in Northern Portugal // Cerebrovasc Dis. 2011. No 32. P. 542-551. DOI: 10.1159/000331473

Markov A.L., Zenchenko T.A., Solonin Y.G., Boiko E.R. Susceptibility Of The Body Functions To Atmospheric And Geomagnetic Factors In Healthy Men Living In Northern Russia // Human Physiology. 2015. No 41 (7). P. 785-788. DOI: $10.1134 /$ S0362119715070130

Mehta A.J., Kloog I., Zanobetti A., Coull B.A., Sparrow D., Vokonas P., Schwartz J. Associations between changes in city and address specific temperature and QT interval: the VA normative aging study // PLoS ONE 2014. No 9 (9). P. 1-9. DOI: 10.1371/journal.pone.0106258

Mostofsky E., Wilker E.H., Schwartz J., Zanobetti A., Gold D.R., Wellenius G.A., et al. Short-term changes in ambient temperature and risk of ischemic stroke // Cerebrovasc Dis Extra. 2014. No 4. P. 9-18. DOI: 10.1159/000357352

Ng C.F., Ueda K., Takeuchi A., Nitta H., Konishi S., Bagrowicz R., Watanabe C., Takami A. Sociogeographic variation in the effects of heat and cold on daily mortality in Japan // J. Epidemiol. 2014. No 24. P. 15-24. DOI: 10.2188/jea.JE20130051

Ozheredov V.A., Chibisov S.M., Blagonravov M.L., Khodorovich N.A., Demurov E.A., Goryachev V.A., Kharlitskaya E.V., Eremina I.S., Meladze Z.A. Influence of Geomagnetic Activity And Earth Weather Changes On Heart Rate And Blood Pressure In Young And Healthy Population//International Journal of Biometeorology. 2017. No 61 (5). P. 921-929. DOI: 10.1007/s00484-016$1272-2$

Patrakeeva V.P., Basova E.E. Effects of Low Temperatures on the Formation of Adaptive Reactions: A Review // International Journal of Biomedicine. 2018. No 8 (2). P. 95-101. DOI: 10.21103/Article8(2)_RA1

Ren C., O'Neill M.S., Park S.K., Sparrow D., Vokonas P., Schwartz J. Ambient temperature, air pollution, and heart rate variability in an aging population // Am J Epidemiol. 2011. No 173 (9). P. 1013-1021. DOI: 10.1093/aje/kwq477

Shaposhnikov D., Revich B., Gurfinkel Y., Naumova E. The influence of meteorological and geomagnetic factors on acute myocardial infarction and brain stroke in Moscow, Russia // Int. J. Biometeorol. 2014. No 58. P. 799. DOI: 10.1007/s00484-013-0660-0

Schwartz J., Samet J.M., Patz J.A. Hospital admissions for heart disease: the effects of temperature and humidity // Epidemiology. 2004. No 15. P. 755-761. DOI: 10.1097/01.ede.0000134875.15919.0f.

Turner L.R., Barnett A.G., Connell D., Tong S. Ambient temperature and cardiorespiratory morbidity: a systematic review and meta-analysis // Epidemiology. 2012. No 23 (4). P. 594-606.

Vencloviene J., Babarskiene R.M., Dobozinskas P., Sakalyte G., Lopatiene K., Mikelionis N. Effects of Weather and Heliophysical Conditions on Emergency Ambulance Calls for Elevated Arterial Blood Pressure // Int. J. Environ. Res. Public Health. 2015. No 12. P. 2622-2638. DOI: 10.3390/ijerph120302622

Vencloviene J., Braziene A. and Dobozinskas P. Short-Term Changes in Weather and Space Weather Conditions and Emergency Ambulance Calls for Elevated Arterial Blood Pressure // Atmosphere. 2018. No 9. P. 114. doi: 10.3390/atmos9030114

Williams S., Nitschke M., Weinstein P., Pisaniello D.L., Parton K.A., Bi P. The impact of summer temperatures and heatwaves on mortality and morbidity in Perth, Australia 1994-2008 // Environ Int. 2012. No 40. P. 33-38. DOI: 10.1016/j.envint.2011.11.011

Wolf K., Schneider A., Breitner S., von Klot S., Meisinger C., Cyrys J. et al. Air temperature and the occurrence of myocardial infarction in Augsburg, Germany // Circulation. 2009. No 120. P. 735-742. DOI: 10.1161/CIRCULATIONAHA.108.815860.

Woodhouse P.R., Khaw K.T., Plummer M. Seasonal variation of blood pressure and its relationship to ambient temperature in an elderly population // J. Hypertens. 1993. No 11. P. 1267-1274.

Ye X., Wolff R., Yu.W., Vaneckova P., Pan X., Tong S. Ambient temperature and morbidity: a review of epidemiological evidence // Environ Health Perspect. 2012. No 120. P. 19-28. DOI: 10.1289/ehp.1003198

Zhang Y., Yu Y., Peng M., Meng R., Hu K., Yu C. Temporal and seasonal variations of mortality burden associated with hourly temperature variability: a nationwide investigation in England and Wales // Environ Int. 2018. No 115. P. 325-333. DOI: 10.1016/j.envint.2018.03.036

Zhao H-Y., Cheng J-M. Associations between ambient temperature and acute myocardial infarction // Open Med. 2019. No 14. P. 14-21. DOI: $10.1515 /$ med-2019-0003

\section{Авторы:}

Зенченко Татьяна Александровна, доктор биологических наук, старший научный сотрудник, лаборатория физической бихимии, Институт теоретической и экспериментальной биофизики РАН, Пущино, Московская область, Россия; старший научный сотрудник, отдел № 54, Институт космических исследований РАН, Москва, Россия.

E-mail: zench@mail.ru

Бреус Тамара Константиновна, доктор физико-математических наук, главный научный сотрудник, отдел № 53, Институт космических исследований РАН, Москва, Россия.

E-mail: breus36@mail.ru 


\author{
T.A. Zenchenko ${ }^{1,2}$, T.K. Breus ${ }^{1}$ \\ ${ }^{1}$ Space Research Institute, Russian Academy of Sciences, Moscow, Russia \\ ${ }^{2}$ Institute of Theoretical and Experimental Biophysics, Russian Academy of Sciences, Pushchino, Moscow region, Russia
}

\title{
POTENTIAL IMPACTS OF WEATHER AND CLIMATE SLOW VARIATIONS ON HUMAN HEALTH AND WELLNESS. CURRENT PERSPECTIVES
}

An analysis of modern population studies of the effect of variations in atmospheric factors on people's health and morbidity in different regions of the world is carried out. This analysis showed the relativity, non-linearity, non-monotonicity of the meteorological effect, the existence of a significant time lag in its development, as well as the dependence of the degree of its manifestation from the season of the year and the geographical location of the place of residence. The severity of the meteorological effect depends on the person's age and gender, possibly race, nutrition, social status, lifestyle.

Most of the works recognize atmospheric temperature, its current values and the amplitude of variations as the most biotropic factor.

It is not correct to talk about the nature of the meteorological effect in relation to "cardiovascular diseases" in general, since the profiles of the relative risk of developing diseases fundamentally depend on specific concomitant nosology.

However, there is another problem: the difference in individual sensitivity to meteorological factors, as a result of which participants in a single group of patients can react differently to the action of the same factor, up to the difference in the sign of the bioeffect.

We also summed up the results of publications on an individual approach to the analysis of healthy volunteers of different latitudes, conducted over 15 years, which showed that:

- in high and middle latitudes, the dominant factor affecting $60-90 \%$ of the population is atmospheric temperature; in southern latitudes - the temperature and atmospheric pressure;

- the sign of correlation with temperature in the northern latitudes was always negative, in the middle and southern latitudes - both signs were found;

- the sign of correlation of blood pressure with temperature in one person may vary in the ranges above and below zero degrees;

- it is shown that one of the atmospheric biotropic factors may be the partial oxygen content in the air;

- two different-direction types of individual response of blood pressure indicators to changes in air temperature were obtained in the region above zero degrees.

The existence of these two types explains why the probability of detecting a statistically significant meteorological effect for healthy people by the average group blood pressure values is practically zero everywhere except in sufficiently northern latitudes.

So the population-based approach has a number of methodological limitations that can be largely overcome by adding an individual approach to it.

Keywords: biometeorology, meteorological sensitivity, population statistics, individual approach, blood pressure, cardiovascular system.

\section{References}

Andronova T.I., Deryapa N.R., Solomatin A.P. Geliometeotropnyye reaktsii zdorovogo i bol'nogo cheloveka [Heliometeotropic reactions of a healthy and sick person]. Leningrad: Medicine, 1982. 247 p. In Russian

Assman D. Chuvstvitel'nost' cheloveka $k$ pogod [Human sensitivity to weather]. Leningrad: Hydrometeoizdat, 1966. $247 \mathrm{p}$. In Russian

Breus T.K., Gurfinkel Y.I., Zenchenko T.A., Ozheredov V.A. Comparative analysis of different vascular tone sensitivity parameters to meteorological and geomagnetic factors// Izvestiya, Atmos Ocean Phys. 2010. 46(8). pp.965-972 https://doi.org/10.1134/S0001433810080050

Breus T.K., Bingi V.N., Petrukovich A.A. The magnetic factor of solar-terrestrial connections and its effect on humans; physical problems and prospects // Physics-Uspekhi, 2016. 186(5). pp. 568-576.

Varlamova N.G., Zenchenko T.A., Boyko E.R. Annual blood pressure dynamics and weather sensitivity in women // Therapeutic Archive. 2017. 89(12). pp. 56-63. DOI: 10.17116/TERARKH2017891256-63

Zenchenko T.A., Tsagareishvili E.V., Oshchepkova E.V., Rogoza A.N., Breus T.K. K voprosam vliyaniya geomagnitnoy i meteorologicheskoy aktivnosti na bol'nykh arterial'noy gipertoniyey [To the questions of the influence of geomagnetic and meteorological activity on patients with arterial hypertension] // Clinical Medicine. 2007. No. 1, pp. 31-35. In Russian

Zenchenko T.A., Tsandekov P.A., Grigoriev P.E., Merzlyi A.M., Zenchenko K.I., Khorseva N.I., Grigal P.P. Issledovaniye kharaktera svyazey fiziologicheskikh $i$ psikhofiziologicheskikh pokazateley organizma s meteorologicheskimi i geomagnitnymi faktorami [Pattern of relations between physiological and psychophysiological parameters of human organism and geomagnetic and meteorological factors] // Geophysical Processes and Biosphere, 2008. V. 7. N 3. pp. 25-36. In Russian

Zenchenko T.A., Rekhtina A.G., Khorseva N.I., Zaslavskaya R.M., Breus T.K. Comparative Analysis of Sensitivity of Different Hemodynamics Indices Of Healthy People To Atmospheric Factors At Middle Latitudes. Geophysical Processes and Biosphere, 2009. T. 8. №4. pp. 61-76. In Russian

Zenchenko T.A., Merzly A.M., Solonin Yu.G. Sravneniye sluchayev individual'noy meteochuvstvitel'nosti cheloveka v ekstremal'nykh usloviyakh zimy severnykh $i$ srednikh shirot [Comparison of individual weather sensitivity of a person in extreme winter conditions of northern and middle latitudes] // Human Ecology. 2011. № 11. pp. 3-13. In Russian)

http://www.iki.rssi.ru/books/2013breus2.pdf (Zenchenko T.A. Metod posledovatel'nykh priblizheniy $v$ zadache issledovaniya mekhanizma individual'nykh geliometeotropnykh reaktsiy [Method of successive approximations in the task of studying the mechanism of individual heliometeotropic reactions]. // Proceedings of the international conference "The influence of space weather on human health in space and on Earth". Moscow. 2012, June 4-7, pp. 633-648. http://www.iki.rssi.ru/books/2013breus2.pdf. In Russian

Zenchenko T.A., Skavulyak A.N., Khorseva N.I., Breus T.K. Characteristics of individual reactions of the cardiovascular system of healthy people to changes in meteorological factors in a wide temperature range. // Izvestiya, Atmospheric and Oceanic Physics. 2013. 49(8). pp. 784-798. DOI: 10.1134/S0001433813080094 
Zenchenko T.A., Varlamova N.G. Hemodynamic Response Characteristics Of Healthy People To Changes In Meteorological And Geomagnetic Factors In The North // Izvestiya. Atmospheric and Oceanic Physics. 2015. 51(8).pp. 858-870. DOI: $10.1134 / \mathrm{S} 0001433815080071$

Kuznetsova E.A., Sirota N.P., Zenchenko T.A. Changes in the Level of DNA in Mouse Cell Induced by Atmospheric Factors // Biophysics. 2019. 64(3). pp. 349-357 DOI: 10.1134/S0006350919030114

Ovcharova V.F. Opredeleniye soderzhaniya kisloroda $v$ atmosfernom vozdukhe na osnove meteorologicheskikh parametrov (davleniya, temperatury, vlazhnosti) s tsel'yu prognozirovaniya gipoksicheskogo effekta atmosfery [Determination of oxygen content in the air based on meteorological parameters (pressure, temperature, humidity) in order to predict the hypoxic effect of the atmosphere] // Questions of balneology, physiotherapy and physiotherapy. 1981. N. 2. pp. 29-34. In Russian

Ozheredov V.A., Breus T.K., Gurfinkel Y.I., Revich D.A., Mitrofanova T.A. Influence of some weather factors and geomagnetic activity on the development of severe cardiological pathologies // Biophysics. 2010. 55(1). pp. 110-119. https://doi.org/10.1134/S0006350910010185

Poskotinova L.V., Grigoriev P.E. Zavisimost' tipologicheskikh osobennostey vegetativnykh reaktsiy zdorovykh lits ot fonovykh pokazateley [The Dependence Of Typological Autonomic Features Reactions Of Healthy Persons On Background Helio-Meteofactors] // Human Ecology. 2008. N 5. pp. 3-8. In Russian

Shepoval'nikov V.N., Soroko S.I. Meteochuvstvitel'nost' cheloveka / otv. red. V. A.Yakovlev; AN Respubliki Kyrgyzstan, In-t fiziologii i eksperimental'noi patologii vysokogor'ya. Bishkek: Ilim, 1992. 247 p. In Russian

Alperovitch A., Lacombe J.M., Hanon O., Dartigues J.F., Ritchie K., Ducimetière P., Tzourio C. Relationship between blood pressure and outdoor temperature in a large sample of elderly individuals: The Three-City study // Arch. Intern. Med. 2009. 169. pp. 75-80. DOI: 10.1001/archinternmed.2008.512

Armstrong B. Models for the relationship between ambient temperature and daily mortality // Epidemiology. 2006. 17(6). pp. 624631. DOI: 10.1097/01.ede.0000239732.50999.8f

Azcárate T., Mendoza B. Influence of geomagnetic activity and atmospheric pressure in hypertensive adults // Int J Biometeorol. 2017. 61. P. 1585-1592. DOI 10.1007/s00484-017-1337-x

Basu R. High ambient temperature and mortality: a review of epidemiologic studies from 2001 to 2008 // Environ Health. 2009.8. pp. 40

Basu R., Pearson D., Malig B., Broadwin R., Green R. The effect of high ambient temperature on emergency room visits // Epidemiology. 2012. 23. pp. 813-820. doi:10.1097/EDE.0b013e31826b7f97.

Bayentin L., El Adlouni S., Ouarda T.B., Gosselin P., Doyon B., Chebana F. Spatial variability of climate effects on ischemic heart disease hospitalization rates for the period 1989-2006 in Quebec, Canada // Int J Health Geogr. 2010. 9. pp.5 https://doi.org/10.1186/1476-072X-9-5

Berginer V.M., Goldsmith J., Batz U., Vardi H., Shapiro Y. Clustering of strokes in association with meteorologic factors in the Negev desert of Israel: 1981-1983 // Stroke. 1989. 20. pp. 65-69 https://doi.org/10.1161/01.STR.20.1.65

Bhaskaran K., Hajat S., Haines A., Herrett E., Wilkinson P., Smeeth L. Effects of ambient temperature on the incidence of myocardial infarction // Heart. 2009. 95. pp. 1760-1769 http://dx.doi.org/10.1136/hrt.2009.175000

Casas A.L.F., Santos G.M.D., Chiocheti N.B., de Andrade M. Effects of Temperature Variation on the Human Cardiovascular System: A Systematic Review // In Climate Change and Health. Climate Change Management; Leal Filho,W., Azeiteiro, U., Alves, F., Eds.; 2016. Springer: Cham, Switzerland

Comelli I., Ferro J., Lippi G., Comelli D., Sartori E., Cervellin G. Incidence of acute-onset atrial fibrillation correlates with air temperature. Results of a nine-year survey // J Epidemiol Glob Health. 2014. 4. pp. 151-157 https://doi.org/10.1016/j.jegh.2013.12.003

Čulič V., Eterovič D., Mirič D., Giunio L., Lukin A., Fabijanič D. Triggering of ventricular tachycardia by meteorologic and emotional stress: protective effect of b-blockers and anxiolytics in men and elderly // Am J Epidemiol. 2004. 160. pp. 1047-1058 https://doi.org/10.1093/aje/kwh335

Danet S., Richard F., Montaye M., Beauchant S., Lemaire B., Graux C., Cottel D., Marécaux N., Amouyel P. Unhealthy effects of atmospheric temperature and pressure on the occurrence of myocardial infarction and coronary deaths. A 10-Year Survey: The LilleWorld Health Organization MONICA Project (Monitoring trends and determinants in cardiovascular disease) // Circulation. 1999. 100, e1-e7. doi:10.1161/01.CIR.100.1.e1

Davis R.E., Hondula D.M., Sharif H. Examining the diurnal temperature range enigma: why is human health related to the daily change in temperature? // Int J Biometeorol. 2020. 64. pp. 397-407 https://doi.org/10.1007/s00484-019-01825-8

Delyukov A., Gorgo Y., Cornélissen G. [et.al.] Natural environmental associations in a 50-day human electrocardiogram // Int J Biometeorol. 2001. 45(2). pp. 90 -99 https://doi.org/10.1007/s004840100086

Dockery D.W., Pope C.A. 3rd, Kanner R.E., Villegas M.G., Schwartz J. Daily changes in oxygen saturation and pulse rate associated with particulate air pollution and barometric pressure // Res. Rep. Health Eff. Inst. 1999. 83. pp. 1-19. PMID: 10192116

Ebi K.L., Exuzides K.A., Lau E., Kelsh M., Barnston A. Weather changes associated with hospitalizations for cardiovascular diseases and stroke in California, 1983-1998 // Int J Biometeorol. 2004. 49. pp. 48-58 https://doi.org/10.1007/s00484-004-0207-5

Feigin V.L., Nikitin Y.P., Bots M.L., Vinogradova T.E., Grobbee D.E. A population-based study of the associations of stroke occurrence with weather parameters in Siberia, Russia (1982-92) // Eur J Neurol. 2000. 7. pp. 171-178. https://doi.org/10.1046/j.14681331.2000.00016.x

Głuszak A., Kocoń S., Zuk K., Aljabali P., Gluza A., Siwek K. Episodes of atrial fibrillation and meteorological conditions // Kardiol Pol. 2008. 66. pp. 958-963 https://www.mp.pl/kardiologiapolska/issue/article/10318

Gurfinkel Y.I., Breus T.K., Zenchenko T.A., Ozheredov V.A. Investigation of the effect of ambient temperature and geomagnetic activity on the vascular parameters of healthy volunteers // Open J Biophys. 2012. 2. pp. 46-55. doi:10.4236/ojbiphy.2012.22007

Halonen J.I., Zanobetti A., Sparrow D., Vokonas P.S., Schwartz J. Relationship between outdoor temperature and blood pressure // Occup. Environ. Med. 2011. 68. pp. 296-301. DOI: 10.1136/oem.2010.056507

Hong Y.C., Kim H., Oh S.Y., Lim Y.H., Kim S.Y., Yoon H.J. et al Association of cold ambient temperature and cardiovascular markers // Sci Total Environ. 2012. 435-436. pp. 74-79. https://doi.org/10.1016/j.scitotenv.2012.02.070

Houck P., Lethen J., Riggs M., Gantt S., Dehmer G. Relation of atmospheric pressure changes and the occurrences of acute myocardial infarction and stroke // Am J Cardiol. 2005. 96. pp. 45-51. doi:10.1016/j.amjcard.2005.02.042 
Jie Y., Houjin H., Mengxue Y., Wei Q., Jie X. A time series analysis of meteorological factors and hospital outpatient admissions for cardiovascular disease in the Northern district of Guizhou Province, China // Braz J Med Biol Res. 2014. 47(8). pp. 689-696. DOI: 10.1590/1414-431X2014424

Kenney W.L., Craighead D.H., Alexander L.M. Heat waves, aging, and human cardiovascular health // Med Sci Sports Exerc. 2014. 46(10). pp. 1891-1899. doi: 10.1249/MSS.0000000000000325

Kulakov I.V., Nasonova E.V. The specific features of circadian blood pressure variations in patients with hypertensive disease in different types of weather // Klin. Med. 2004. 82. pp. 24-27. PMID: 15468719

Lavigne E., Gasparrini A., Wang X., Yagouti A., Fleury M.D., Cakmak S. Extreme ambient temperatures and cardiorespiratory emergency room visits: assessing risk by comorbid health conditions in a time series study // Environ Health. 2014. 13. pp. 5 https://doi.org/10.1186/1476-069X-13-5

Lin S., Luo M., Walker R.J., Liu X., Hwang S.A., Chinery R. Extreme high temperatures and hospital admissions for respiratory and cardiovascular diseases // Epidemiology. 2009. 20. pp. 738-746. doi: 10.1097/EDE.0b013e3181ad5522

Linares C., Díaz J. Impact of high temperatures on hospital admissions: comparative analysis with previous studies about mortality (Madrid) // The European Journal of Public Health. 2008. 18(3). pp. 317-322. https://doi.org/10.1093/eurpub/ckm108

Madaniyazi L., Guo Y., Williams G. et al. The nonlinear association between outdoor temperature and cholesterol levels, with modifying effect of individual characteristics and behaviors // Int J Biometeorol. 2020. 64. pp. 367-375. https://doi.org/10.1007/s00484019-01816-9

Magalhães R., Silva M.C., Corrêia M., Bailey T. Are stroke occurrence and outcome related to weather parameters? Results from a population-based study in Northern Portugal // Cerebrovasc Dis. 2011. 32. pp. 542-551 https://doi.org/10.1159/000331473

Markov A.L., Zenchenko T.A., Solonin Y.G., Boiko E.R. Susceptibility Of The Body Functions To Atmospheric And Geomagnetic Factors In Healthy Men Living In Northern Russia. // Human Physiology. 2015. 41(7). pp. 785-788. DOI: 10.1134/S0362119715070130

Mehta A.J., Kloog I., Zanobetti A., Coull B.A., Sparrow D., Vokonas P., Schwartz J. Associations between changes in city and address specific temperature and QT interval: the VA normative aging study // PLoS ONE 2014. 9(9). pp. 1-9. doi: 10.1371/journal.pone. 0106258

Mostofsky E., Wilker E.H., Schwartz J., Zanobetti A., Gold D.R., Wellenius G.A., et al. Short-term changes in ambient temperature and risk of ischemic stroke // Cerebrovasc Dis Extra. 2014. 4. pp. 9-18. doi: 10.1159/000357352

Ng C.F., Ueda K., Takeuchi A., Nitta H., Konishi S., Bagrowicz R., et al. Sociogeographic variation in the effects of heat and cold on daily mortality in Japan // J Epidemiol. 2014. 24. pp. 15-24. doi: 10.2188/jea.JE20130051

Ozheredov V.A., Chibisov S.M., Blagonravov M.L., Khodorovich N.A., Demurov E.A., Goryachev V.A., Kharlitskaya E.V., Eremina I.S., Meladze Z.A. Influence of Geomagnetic Activity And Earth Weather Changes On Heart Rate And Blood Pressure In Young And Healthy Population//International Journal of Biometeorology. 2017. 61(5). pp. 921-929. DOI: 10.1007/s00484-016-1272-2

Patrakeeva V.P., Basova E.E. Effects of Low Temperatures on the Formation of Adaptive Reactions: A Review // International Journal of Biomedicine. 2018. 8(2). pp. 95-101. DOI: 10.21103/Article8(2)_RA1

Ren C., O'Neill M.S., Park S.K., Sparrow D., Vokonas P., Schwartz J. Ambient temperature, air pollution, and heart rate variability in an aging population // Am J Epidemiol. 2011. 173(9). pp. 1013-1021. DOI: 10.1093/aje/kwq477

Shaposhnikov D., Revich B., Gurfinkel Y., Naumova E. The influence of meteorological and geomagnetic factors on acute myocardial infarction and brain stroke in Moscow, Russia // Int J Biometeorol. 2014. 58. pp. 799. doi:10.1007/s00484-013-0660-0

Schwartz J., Samet J.M., Patz J.A. Hospital admissions for heart disease: the effects of temperature and humidity //Epidemiology. 2004. 15. pp. 755-761. doi: 10.1097/01.ede.0000134875.15919.0f.

Turner L.R., Barnett A.G., Connell D., Tong S. Ambient temperature and cardiorespiratory morbidity: a systematic review and meta-analysis // Epidemiology. 2012. 23(4). pp. 594-606 www.jstor.org/stable/23214303

Vencloviene J., Babarskiene R.M., Dobozinskas P., Sakalyte G., Lopatiene K., Mikelionis N. Effects of Weather and Heliophysical Conditions on Emergency Ambulance Calls for Elevated Arterial Blood Pressure // Int. J. Environ. Res. Public Health. 2015.12. pp. 2622-2638; doi:10.3390/ijerph120302622

Vencloviene J., Braziene A. and Dobozinskas P. Short-Term Changes in Weather and Space Weather Conditions and Emergency Ambulance Calls for Elevated Arterial Blood Pressure // Atmosphere. 2018. 9. pp. 114. doi:10.3390/atmos9030114

Williams S., Nitschke M., Weinstein P., Pisaniello D.L., Parton K.A., Bi P. The impact of summer temperatures and heatwaves on mortality and morbidity in Perth, Australia 1994-2008 // Environ Int. 2012. 40. pp. 33-38. https://doi.org/10.1016/j.envint.2011.11.011

Wolf K., Schneider A., Breitner S., von Klot S., Meisinger C., Cyrys J., et al. Air temperature and the occurrence of myocardial infarction in Augsburg, Germany // Circulation. 2009. 120. pp. 735-742. doi:10.1161/CIRCULATIONAHA.108.815860.

Woodhouse P.R., Khaw K.T., Plummer M. Seasonal variation of blood pressure and its relationship to ambient temperature in an elderly population // J. Hypertens. 1993. 11. pp. 1267-1274. PMID: 8301109

Ye X., Wolff R., Yu W., Vaneckova P., Pan X., Tong S. Ambient temperature and morbidity: a review of epidemiological evidence // Environ Health Perspect. 2012. 120. pp. 19-28. https://doi.org/10.1289/ehp.1003198

Zhang Y., Yu Y., Peng M., Meng R., Hu K., Yu C. Temporal and seasonal variations of mortality burden associated with hourly temperature variability: a nationwide investigation in England and Wales // Environ Int. 2018. 115. pp.325-333. https://doi.org/10.1016/j.envint.2018.03.036

Zhao H-Y., Cheng J-M. Associations between ambient temperature and acute myocardial infarction // Open Med. 2019. 14. pp. 1421. https://doi.org/10.1515/med-2019-0003

\section{Authors:}

Zenchenko Tatyana A., Dr. Sci. (Biology), Senior Researcher, Institute of Theoretical and Experimental Biophysics, Russian Academy of Sciences, Pushchino, Moscow Region, Russia; Senior Researcher, Space Research Institute, Russian Academy of Sciences, Moscow, Russia.

E-mail: zench@mail.ru

Breus Tamara K., Dr. Sci. (Phys.-Math.), Chief Researcher, Space Research Institute of the Russian Academy of Sciences, Moscow, Russia.

E-mail: breus36@mail.ru 